\title{
Symbolic computation of exact solutions for fractional differential-difference equation models
}

\author{
İsmail Aslan \\ Department of Mathematics, İzmir Institute of Technology \\ Urla, İzmir 35430, Turkey \\ ismailaslan@iyte.edu.tr
}

Received: July 30, 2013 / Revised: June 9, 2014 / Published online: November 14, 2014

\begin{abstract}
The aim of the present study is to extend the $\left(G^{\prime} / G\right)$-expansion method to fractional differential-difference equations of rational type. Particular time-fractional models are considered to show the strength of the method. Three types of exact solutions are observed: hyperbolic, trigonometric and rational. Exact solutions in terms of topological solitons and singular periodic functions are also obtained. As far as we are aware, our results have not been published elsewhere previously.
\end{abstract}

Keywords: fractional calculus, differential-difference equation, $\left(G^{\prime} / G\right)$-expansion method.

\section{Introduction}

Fractional calculus is the study of differentiation and integration of arbitrary order and is used in many areas such as fluid dynamics, turbulence, image processing, finance, nonlinear control theory, astrophysics, stochastic dynamical systems, plasma physics, nonlinear biological systems, etc. [16, 26, 32]. Fractional differential equations (FDEs) are regarded as models of physical systems. Hence, various analytical and numerical techniques have been developed for solving FDEs. These methods include Adomian decomposition method [6], differential transform method [30], finite element method [12], finite difference method [4], variational iteration method [31], homotopy perturbation method [9], fractional sub-equation method [39] and first integral method [19]. Different definitions of fractional derivatives are used. The Caputo fractional derivative, the Riemann-Liouville fractional derivative, the Sonin-Letnikov fractional derivative, the Riesz fractional derivative, the Marchaud fractional derivative, the Weyl fractional derivative, the Grünwald-Letnikov fractional derivative are some examples of these definitions. In addition, Kolwankar-Gangal derivative [17], the Cresson's derivative [3] and the Jumarie's modified Riemann-Liouville derivative [13], which are the local versions of fractional derivatives, are also defined in the related literature.

On the other hand, differential-difference equations (DDEs), lattices or networks, are also used in the physical, chemical, biological, social, medical and engineering sciences. 
To name a few; wave phenomena in fluids, chemical reactions, particle vibrations in lattices, currents in electrical networks, pulses in biological chains. So far, various lattices such as the Ablowitz-Ladik lattice, the Toda lattice, the Volterra lattice, the discrete (modified) KdV equation, the Suris lattice (see [33]) have been discovered by researchers since the original work of Fermi, Pasta and Ulam [7]. Accordingly, developing analyti$\mathrm{cal} /$ numerical methods for solving DDEs has become an important issue. The Casoratian technique [25], the Hirota's bilinear method [11], the exp-function method [5], the homotopy perturbation method [40], the ADM-Padé technique [36], the $\left(G^{\prime} / G\right)$-expansion method [37] are the most commonly used ones. In the meantime, DDEs are considered to be hybrid systems as well because the spatial variable is discrete while the time variable is continuous in a DDE.

Recently, He et al. [10] have suggested the reformulation of DDEs in the context of fractional derivatives. In addition, they have pointed out the new directions in nonlinear science to solve differential equations by proposing three standard variational iterations, integro-differential equations, FDEs, fractal differential equations, DDEs and fractal DDEs. The present paper aims to investigate fractional DDEs of the form

$$
D_{t}^{\alpha} u_{n}=R\left(u_{n-1}, u_{n}, u_{n+1}\right), \quad 0<\alpha \leqslant 1,
$$

where $D_{t}^{\alpha}$ is the Jumarie's modified Riemann-Liouville derivative (in time) of order $\alpha$, $R$ is a rational function of its arguments, $n \in \mathbb{Z}, u_{n}(t)=u(n, t)$ is the displacement of the $n$th particle from the equilibrium position. Accordingly, the following particular time-fractional DDEs will be analyzed by means of the $\left(G^{\prime} / G\right)$-expansion method [38]:

$$
\begin{aligned}
D_{t}^{\alpha} u_{n} & =\frac{u_{n-1}-u_{n+1}+2 u_{n-1} u_{n}+2 u_{n} u_{n+1}-2 u_{n-1} u_{n+1}-2 u_{n}^{2}}{1+u_{n-1}-u_{n+1}}, \quad 0<\alpha \leqslant 1, \\
D_{t}^{\alpha} u_{n} & =\frac{2\left(u_{n-1}-u_{n+1}\right) u_{n}^{2}\left(1-u_{n}^{2}\right)}{\left(u_{n-1}+u_{n}\right)\left(u_{n}+u_{n+1}\right)}, \quad 0<\alpha \leqslant 1 .
\end{aligned}
$$

The $\left(G^{\prime} / G\right)$-expansion method has been shown to be a powerful analytical tool for different kinds of nonlinear problems. It is a promising and evolving method. Indeed, the $\left(G^{\prime} / G\right)$-expansion method can be applied to FDEs in a straightforward manner (see $[2,8])$. In the mean time, it is important to note that the case $\alpha=1$ of Eqs. (2) and (3) are considered in [27] and [28], respectively.

This paper is organized as follows. In Section 2, the preliminaries are presented. In Section 3, the method of the study is explained. In Sections 4 and 5, time-fractional DDEs (2) and (3) are analyzed. Finally, Section 6 is devoted to concluding remarks.

\section{Preliminaries}

In this section, a brief background on the definition of the Jumarie's modified RiemannLiouville derivative is presented. To this end, let $f: \mathbb{R} \rightarrow \mathbb{R}, t \rightarrow f(t)$, denote a continuous (but not necessarily differentiable) function. The Jumarie's modified Riemann- 
Liouville derivative of order $\alpha$ is defined as follows:

$$
\begin{aligned}
& D_{t}^{\alpha} f(t)=\frac{1}{\Gamma(-\alpha)} \int_{0}^{t}(t-\xi)^{-\alpha-1}(f(\xi)-f(0)) \mathrm{d} \xi, \quad \alpha<0, \\
& D_{t}^{\alpha} f(t)=\frac{1}{\Gamma(1-\alpha)} \frac{\mathrm{d}}{\mathrm{d} t} \int_{0}^{t}(t-\xi)^{-\alpha}(f(\xi)-f(0)) \mathrm{d} \xi, \quad 0<\alpha<1, \\
& D_{t}^{\alpha} f(t)=\left(f^{(\alpha-n)}(t)\right)^{(n)}, \quad n \leqslant \alpha<n+1, n \geqslant 1 .
\end{aligned}
$$

This fractional derivative has been successfully implemented to fractional variational calculus [35], probability calculus [14] and fractional Laplace problems [15]. Moreover, the following properties hold [13]:

$$
\begin{gathered}
D_{t}^{\alpha} t^{\gamma}=\frac{\Gamma(1+\gamma)}{\Gamma(1+\gamma-\alpha)} t^{\gamma-\alpha}, \quad \gamma>0, \\
D_{t}^{\alpha}(f(t) g(t))=g(t) D_{t}^{\alpha} f(t)+f(t) D_{t}^{\alpha} g(t), \\
D_{t}^{\alpha} f(g(t))=f_{g}^{\prime}(g(t)) D_{t}^{\alpha} g(t)=D_{g}^{\alpha} f(g(t))\left(g_{t}^{\prime}\right)^{\alpha} .
\end{gathered}
$$

These are direct consequences of the equality $\mathrm{d}^{\alpha} x(t)=\Gamma(1+\alpha) \mathrm{d} x(t)$. The Jumarie's modified Riemann-Liouville derivative has many significant properties:

(i) the $\alpha$-order derivative of a constant is zero;

(ii) it can be applied to both differentiable and nondifferentiable functions.

\section{Outline of the $\left(G^{\prime} / G\right)$-expansion method}

According to Li and He [18], FDEs can be converted into ODEs via the fractional complex transformation

$$
\xi=\frac{p x^{\alpha}}{\Gamma(1+\alpha)}+\frac{q y^{\beta}}{\Gamma(1+\beta)}+\frac{r t^{\gamma}}{\Gamma(1+\gamma)}, \quad 0<\alpha \leqslant 1,0<\beta \leqslant 1,0<\gamma \leqslant 1,
$$

where $p, q$ and $r$ are constants to be determined. Now, to illustrate the basic idea of the method, we consider a system of $M$ fractional DDEs in the form

$$
\begin{gathered}
\Delta\left(\mathbf{u}_{\mathbf{n}+\mathbf{p}_{1}}(\mathbf{x}), \ldots, \mathbf{u}_{\mathbf{n}+\mathbf{p}_{k}}(\mathbf{x}), \ldots, \mathbf{u}_{\mathbf{n}+\mathbf{p}_{1}}^{\alpha}(\mathbf{x}), \ldots, \mathbf{u}_{\mathbf{n}+\mathbf{p}_{k}}^{\alpha}(\mathbf{x}), \ldots,\right. \\
\left.\quad \mathbf{u}_{\mathbf{n}+\mathbf{p}_{1}}^{(r \alpha)}(\mathbf{x}), \ldots, \mathbf{u}_{\mathbf{n}+\mathbf{p}_{k}}^{(r \alpha)}(\mathbf{x})\right)=0, \quad 0<\alpha \leqslant 1,
\end{gathered}
$$

where the dependent variable $\mathbf{u}_{\mathbf{n}}$ have $M$ components $u_{i, \mathbf{n}}$ and so do its shifts; the continuous variable $\mathbf{x}$ has $N$ components $x_{i}$; the discrete variable $\mathbf{n}$ has $Q$ components $n_{j}$; the $k$ shift vectors $\mathbf{p}_{i} \in \mathbb{Z}^{Q}$; and $\mathbf{u}^{(r \alpha)}(\mathbf{x})$ denotes the collection of Jumarie's modified 
Riemann-Liouville derivative terms of order $r \alpha$. To search for exact solutions of Eq. (11), the following fractional complex transformation is taken into consideration:

$$
\mathbf{u}_{\mathbf{n}+\mathbf{p}_{s}}(\mathbf{x})=\mathbf{U}_{\mathbf{n}+\mathbf{p}_{s}}\left(\xi_{\mathbf{n}}\right), \quad \xi_{\mathbf{n}}=\sum_{i=1}^{Q} d_{i} n_{i}+\sum_{j=1}^{N} \frac{c_{j}}{\Gamma(1+\alpha)} x_{j}^{\alpha}+\zeta,
$$

where $s=1,2, \ldots, k$. The coefficients $c_{1}, c_{2}, \ldots, c_{N}, d_{1}, d_{2}, \ldots, d_{Q}$ and the phase $\zeta$ are all constants while $\Gamma$ denotes the gamma function. Then Eq. (11) changes into a system of DDEs of integer order in the form

$$
\begin{gathered}
\Delta\left(\mathbf{U}_{\mathbf{n}+\mathbf{p}_{1}}\left(\xi_{\mathbf{n}}\right), \ldots, \mathbf{U}_{\mathbf{n}+\mathbf{p}_{k}}\left(\xi_{\mathbf{n}}\right), \ldots, \mathbf{U}_{\mathbf{n}+\mathbf{p}_{1}}^{\prime}\left(\xi_{\mathbf{n}}\right), \ldots, \mathbf{U}_{\mathbf{n}+\mathbf{p}_{k}}^{\prime}\left(\xi_{\mathbf{n}}\right), \ldots,\right. \\
\mathbf{U}_{\mathbf{n}+\mathbf{p}_{1}}^{(r)}\left(\xi_{\mathbf{n}}\right), \ldots, \mathbf{U}_{\mathbf{n}+\mathbf{p}_{k}}^{(r)}\left(\xi_{\mathbf{n}}\right)=0 .
\end{gathered}
$$

We asume the solution(s) of (13) can be written as a finite power series in $G^{\prime}\left(\xi_{\mathbf{n}}\right) / G\left(\xi_{\mathbf{n}}\right)$ like

$$
\mathbf{U}_{\mathbf{n}}\left(\xi_{\mathbf{n}}\right)=\sum_{l=0}^{m} a_{l}\left(\frac{G^{\prime}\left(\xi_{\mathbf{n}}\right)}{G\left(\xi_{\mathbf{n}}\right)}\right)^{l}, \quad a_{m} \neq 0,
$$

where $m$ is a positive integer, $a_{i}$ 's are constants to be determined, $G\left(\xi_{\mathbf{n}}\right)$ is a solution of the second-order linear ordinary differential equation

$$
G^{\prime \prime}\left(\xi_{\mathbf{n}}\right)+\lambda G^{\prime}\left(\xi_{\mathbf{n}}\right)+\mu G\left(\xi_{\mathbf{n}}\right)=0,
$$

where $\lambda$ and $\mu$ are the arbitrary parameters and prime denotes derivative with respect to $\xi_{\mathbf{n}}$. The general solution of Eq. (15) is well known. Thus, the following three cases hold:

$$
\begin{aligned}
& \frac{G^{\prime}\left(\xi_{\mathbf{n}}\right)}{G\left(\xi_{\mathbf{n}}\right)}=-\frac{\lambda}{2}+\frac{\sqrt{\lambda^{2}-4 \mu}}{2} \frac{C_{1} \cosh \left(\frac{\sqrt{\lambda^{2}-4 \mu}}{2} \xi_{\mathbf{n}}\right)+C_{2} \sinh \left(\frac{\sqrt{\lambda^{2}-4 \mu}}{2} \xi_{\mathbf{n}}\right)}{C_{1} \sinh \left(\frac{\sqrt{\lambda^{2}-4 \mu}}{2} \xi_{\mathbf{n}}\right)+C_{2} \cosh \left(\frac{\sqrt{\lambda^{2}-4 \mu}}{2} \xi_{\mathbf{n}}\right)} \\
& \lambda^{2}-4 \mu>0, \\
& \frac{G^{\prime}\left(\xi_{\mathbf{n}}\right)}{G\left(\xi_{\mathbf{n}}\right)}=-\frac{\lambda}{2}+\frac{\sqrt{4 \mu-\lambda^{2}}}{2} \frac{-C_{1} \sin \left(\frac{\sqrt{4 \mu-\lambda^{2}}}{2} \xi_{\mathbf{n}}\right)+C_{2} \cos \left(\frac{\sqrt{4 \mu-\lambda^{2}}}{2} \xi_{\mathbf{n}}\right)}{C_{1} \cos \left(\frac{\sqrt{4 \mu-\lambda^{2}}}{2} \xi_{\mathbf{n}}\right)+C_{2} \sin \left(\frac{\sqrt{4 \mu-\lambda^{2}}}{2} \xi_{\mathbf{n}}\right)} \\
& \quad \lambda^{2}-4 \mu<0, \\
& \frac{G^{\prime}\left(\xi_{\mathbf{n}}\right)}{G\left(\xi_{\mathbf{n}}\right)}=-\frac{\lambda}{2}+\frac{C_{1}}{C_{1} \xi_{\mathbf{n}}}+C_{2}, \quad \lambda^{2}-4 \mu=0
\end{aligned}
$$

where $C_{1}$ and $C_{2}$ are arbitrary constants. Then a straightforward calculation leads to

$$
\xi_{\mathbf{n}+\mathbf{p}_{s}}=\xi_{\mathbf{n}+\varphi_{s}}, \quad \varphi_{s}=p_{s 1} d_{1}+p_{s 2} d_{2}+\cdots+p_{s Q} d_{Q},
$$


where $p_{s j}$ is the $j$ th component of the shift vector $\mathbf{p}_{s}$. Hence, the following expression can be derived using expressions (16)-(19):

$$
\frac{G^{\prime}\left(\xi_{\mathbf{n} \pm \mathbf{p}_{s}}\right)}{G\left(\xi_{\mathbf{n} \pm \mathbf{p}_{s}}\right)}=-\frac{\lambda}{2}+\frac{\frac{\lambda}{2}+\frac{G^{\prime}\left(\xi_{\mathbf{n}}\right)}{G\left(\xi_{\mathbf{n}}\right)} \pm \varepsilon \frac{\sqrt{\delta+\varepsilon\left(\lambda^{2}-4 \mu\right)}}{2} f\left(\frac{\sqrt{\delta+\varepsilon\left(\lambda^{2}-4 \mu\right)}}{2} \varphi_{s}\right)}{1 \pm \frac{2}{\sqrt{\delta+\varepsilon\left(\lambda^{2}-4 \mu\right)}}\left(\frac{G^{\prime}\left(\xi_{\mathbf{n}}\right)}{G\left(\xi_{\mathbf{n}}\right)}+\frac{\lambda}{2}\right) f\left(\frac{\sqrt{\delta+\varepsilon\left(\lambda^{2}-4 \mu\right)}}{2} \varphi_{s}\right)},
$$

where $\varepsilon \in\{0, \pm 1\}$ and $\delta \in\{0,4\}$,

$$
\begin{aligned}
& f\left(\frac{\sqrt{\delta+\varepsilon\left(\lambda^{2}-4 \mu\right)}}{2} \varphi_{s}\right)=\tanh \left(\frac{\sqrt{\lambda^{2}-4 \mu}}{2} \varphi_{s}\right), \quad \varepsilon=1, \\
& \delta=0, \lambda^{2}-4 \mu>0 ; \\
& f\left(\frac{\sqrt{\delta+\varepsilon\left(\lambda^{2}-4 \mu\right)}}{2} \varphi_{s}\right)=\tan \left(\frac{\sqrt{4 \mu-\lambda^{2}}}{2} \varphi_{s}\right), \quad \varepsilon=-1, \\
& \quad \delta=0, \lambda^{2}-4 \mu<0 ; \\
& f\left(\frac{\sqrt{\delta+\varepsilon\left(\lambda^{2}-4 \mu\right)}}{2} \varphi_{s}\right)=\varphi_{s}, \quad \varepsilon=0, \delta=4, \lambda^{2}-4 \mu=0 .
\end{aligned}
$$

As a result, the following uniform shift formula is obtained:

$$
\begin{aligned}
& \mathbf{U}_{\mathbf{n}+\mathbf{p}_{s}}\left(\xi_{\mathbf{n}}\right) \\
& \quad=\sum_{l=0}^{m} a_{l}\left(-\frac{\lambda}{2}+\frac{\frac{\lambda}{2}+\frac{G^{\prime}\left(\xi_{\mathbf{n}}\right)}{G\left(\xi_{\mathbf{n}}\right)} \pm \varepsilon \frac{\left.\sqrt{\delta+\varepsilon\left(\lambda^{2}-4 \mu\right.}\right)}{2} f\left(\frac{\sqrt{\delta+\varepsilon\left(\lambda^{2}-4 \mu\right)}}{2} \varphi_{s}\right)}{1 \pm \frac{2}{\sqrt{\delta+\varepsilon\left(\lambda^{2}-4 \mu\right)}}\left(\frac{G^{\prime}\left(\xi_{\mathbf{n}}\right)}{G\left(\xi_{\mathbf{n}}\right)}+\frac{\lambda}{2}\right) f\left(\frac{\sqrt{\delta+\varepsilon\left(\lambda^{2}-4 \mu\right)}}{2} \varphi_{s}\right)}\right)^{l} .
\end{aligned}
$$

Balancing the highest-order derivative term and the highest order nonlinear term(s) in $\mathbf{U}_{\mathbf{n}}\left(\xi_{\mathbf{n}}\right)$ as in the continuous case, the degree $m$ of (14) and (24) can be easily determined from (13). The leading terms of $\mathbf{U}_{\mathbf{n}+\mathbf{p}_{s}}\left(\mathbf{p}_{s} \neq \mathbf{0}\right)$ will not have any effect on the balancing procedure because $\mathbf{U}_{\mathbf{n}+\mathbf{p}_{s}}$ can be regarded as being of degree zero in $G^{\prime}\left(\xi_{\mathbf{n}}\right) / G\left(\xi_{\mathbf{n}}\right)$. Substituting (14) and (24) together with (15) into (13), equating the coefficients of $\left(G^{\prime}\left(\xi_{\mathbf{n}}\right) / G\left(\xi_{\mathbf{n}}\right)\right)^{l}(l=0,1,2, \ldots)$ to zero, we obtain a system of nonlinear algebraic equations from which the unspecified constants $a_{i}, d_{i}, c_{j}$ and $k$ can be explicitly found. Finally, substituting these results into (14) will lead to varies kind of exact solutions for (11). Indeed, the $\left(G^{\prime} / G\right)$-expansion method is a special application of Ma and Fuchssteiner's expansion approach presented in [20]. To be more clear, assume that $G=G\left(\xi_{\mathbf{n}}\right)$ is a solution of (15). If we let $U=G^{\prime} / G$, then $U^{\prime}=G^{\prime \prime} / G-U^{2}$ and thus (15) becomes $U^{\prime}+U^{2}+\lambda U+\mu=0$ which is a Riccati equation. This equation corresponds to a particular case $\left(a_{0}=-\mu, a_{1}=-\lambda, a_{2}=-1\right)$ of the Riccati equation $v_{\xi}=a_{0}+a_{1} v+a_{2} v^{2}, a_{i} \in R^{1}, a_{2} \neq 0$, which is (39) of the reference [20]. The general solution of the later Riccati equation was given by expressions (40)-(42) for three cases in [20]. Hence, we conclude that our solutions (16)-(18) for three cases are included by solutions (40)-(42) presented in [20]. 
Remark 1. The basic $\left(G^{\prime} / G\right)$-expansion method takes into account the equation $G^{\prime \prime}+$ $\lambda G^{\prime}+\mu G=0$, where $\lambda$ and $\mu$ are arbitrary parameters. However, Aslan [1] has proved that the parameter $\lambda$ can be set to zero without loss of generality. Taking the parameter $\lambda$ as zero is more advantageous than the original one because it minimizes the number of the parameters at the outset. This approach provides equivalent results.

\section{Analysis of Eq. (2)}

To find exact solutions for Eq. (2), let us first make the fractional complex transformation

$$
u_{n}=U_{n}\left(\xi_{n}\right), \quad \xi_{n}=d n+\frac{k}{\Gamma(1+\alpha)} t^{\alpha}+\chi,
$$

where $d$ and $k$ are real parameters to be specified, while $\chi$ denotes the phase shift. On substituting (25) into Eq. (2), the following equation, where prime denotes ordinary derivative with respect to the new independent variable $\xi_{n}$, is obtained:

$$
\begin{aligned}
& k U_{n}^{\prime}\left(1+U_{n-1}-U_{n+1}\right) \\
& \quad-\left(U_{n-1}-U_{n+1}+2 U_{n-1} U_{n}+2 U_{n} U_{n+1}-2 U_{n-1} U_{n+1}-2 U_{n}^{2}\right)=0 .
\end{aligned}
$$

Then our procedure suggests a solution for Eq. (26) in the form

$$
U_{n}=a_{0}+a_{1} \frac{G^{\prime}}{G}, \quad a_{1} \neq 0,
$$

where $G=G\left(\xi_{n}\right)$ satisfies Eq. (15), while $a_{0}$ and $a_{1}$ are arbitrary constants to be determined at the stage of solving the problem.

\subsection{Hyperbolic function solutions}

In case $\mu<0$, first, the following shift formula

$$
U_{n \pm 1}=a_{0}+a_{1}\left(-\frac{\lambda}{2}+\frac{\frac{\lambda}{2}+\frac{G \prime}{G} \pm \frac{\sqrt{\lambda^{2}-4 \mu}}{2} \tanh \left(\frac{\sqrt{\lambda^{2}-4 \mu}}{2} d\right)}{1 \pm \frac{2}{\sqrt{\lambda^{2}-4 \mu}}\left(\frac{\lambda}{2}+\frac{G \prime}{G}\right) \tanh \left(\frac{\sqrt{\lambda^{2}-4 \mu}}{2} d\right)}\right)
$$

is derived in accordance with (24). Then substituting (27) and (28) along with (15) into Eq. (26), clearing the denominator, setting the coefficients of $\left(G^{\prime} / G\right)^{l}(l=0,2,4)$ to zero, a system of nonlinear algebraic equations for $a_{0}, a_{1}, d, k$ and $\mu$ is obtained. A solution of the resulting system is

$$
\lambda=0, \quad a_{0}=a_{0}, \quad a_{1}=\frac{\tanh (2 d \sqrt{-\mu})}{2 \sqrt{-\mu}}, \quad k=-\frac{\sinh (2 d \sqrt{-\mu})}{\sqrt{-\mu}},
$$

which yields a hyperbolic function solution to Eq. (2) in the form

$$
u_{n}(t)=a_{0}+\frac{1}{2} \tanh (2 d \sqrt{-\mu}) \frac{C_{1} \cosh \left(\sqrt{-\mu} \xi_{n}\right)+C_{2} \sinh \left(\sqrt{-\mu} \xi_{n}\right)}{C_{1} \sinh \left(\sqrt{-\mu} \xi_{n}\right)+C_{2} \cosh \left(\sqrt{-\mu} \xi_{n}\right)},
$$


where $\xi_{n}=d n-\sinh (2 d \sqrt{-\mu}) /(\Gamma(1+\alpha) \sqrt{-\mu}) t^{\alpha}+\chi$, while $a_{0}, d, \chi, \mu(<0), C_{1}$ and $\mathrm{C}_{2}$ remain arbitrary.

\subsection{Trigonometric function solutions}

In case $\mu>0$, first, the following shift formula

$$
U_{n \pm 1}=a_{0}+a_{1}\left(-\frac{\lambda}{2}+\frac{\frac{\lambda}{2}+\frac{G \prime}{G} \mp \frac{\sqrt{4 \mu-\lambda^{2}}}{2} \tan \left(\frac{\sqrt{4 \mu-\lambda^{2}}}{2} d\right)}{1 \pm \frac{2}{\sqrt{4 \mu-\lambda^{2}}}\left(\frac{\lambda}{2}+\frac{G \prime}{G}\right) \tan \left(\frac{\sqrt{4 \mu-\lambda^{2}}}{2} d\right)}\right)
$$

is derived in accordance with (24). Then substituting (27) and (31) along with (15) into Eq. (26), clearing the denominator, setting the coefficients of $\left(G^{\prime} / G\right)^{l}(l=0,2,4)$ to zero, a system of nonlinear algebraic equations for $a_{0}, a_{1}, d, k$ and $\mu$ is obtained. A solution of the resulting system is

$$
\lambda=0, \quad a_{0}=a_{0}, \quad a_{1}=\frac{\tan (2 d \sqrt{\mu})}{2 \sqrt{\mu}}, \quad k=-\frac{\sin (2 d \sqrt{\mu})}{\sqrt{\mu}},
$$

which gives a trigonometric function solution to Eq. (2) in the form

$$
u_{n}(t)=a_{0}+\frac{1}{2} \tan (2 d \sqrt{\mu}) \frac{-C_{1} \sin \left(\sqrt{\mu} \xi_{n}\right)+C_{2} \cos \left(\sqrt{\mu} \xi_{n}\right)}{C_{1} \cos \left(\sqrt{\mu} \xi_{n}\right)+C_{2} \sin \left(\sqrt{\mu} \xi_{n}\right)},
$$

where $\xi_{n}=d n-\sin (2 d \sqrt{\mu}) /(\Gamma(1+\alpha) \sqrt{\mu}) t^{\alpha}+\chi$, while $a_{0}, d, \chi, \mu(>0), C_{1}$ and $C_{2}$ remain arbitrary.

\subsection{Rational function solutions}

In case $\mu=0$, first, the following shift formula

$$
U_{n \pm 1}=a_{0}+a_{1}\left(-\frac{\lambda}{2}+\frac{\frac{G \prime}{G}+\frac{\lambda}{2}}{1 \pm\left(\frac{G \prime}{G}+\frac{\lambda}{2}\right) d}\right)
$$

is derived in accordance with (24). Then substituting (27) and (34) along with (15) into Eq. (26), clearing the denominator, setting the coefficients of $\left(G^{\prime} / G\right)^{l}(l=2,4)$ to zero, a system of nonlinear algebraic equations for $a_{0}, a_{1}, d$ and $k$ is obtained. A solution of the resulting system is

$$
\lambda=0, \quad a_{0}=a_{0}, \quad a_{1}=d, \quad k=-2 d,
$$

which yields a rational function solution to Eq. (2) in the form

$$
u_{n}(t)=a_{0}+d \frac{C_{1}}{C_{1}\left(d n-\frac{2 d}{\Gamma(1+\alpha)} t^{\alpha}+\chi\right)+C_{2}},
$$

where $a_{0}, d, \chi, C_{1}$ and $C_{2}$ remain arbitrary. 
Remark 2. Obviously, some special solutions for Eq. (2) can be constructed by assigning special values to the arbitrary parameters $C_{1}$ and $C_{2}$. For example, setting $C_{1}=0$ and $C_{2} \neq 0$ or $C_{1} \neq 0$ and $C_{2}=0$ in (30), respectively, provides formal solitary wave solutions to Eq. (2) in the form

$$
\begin{aligned}
& u_{n}(t)=a_{0}+\frac{1}{2} \tanh (2 d \sqrt{-\mu}) \tanh \left(\sqrt{-\mu}\left(d n-\frac{\sinh (2 d \sqrt{-\mu})}{\Gamma(1+\alpha) \sqrt{-\mu}} t^{\alpha}+\chi\right)\right), \\
& u_{n}(t)=a_{0}+\frac{1}{2} \tanh (2 d \sqrt{-\mu}) \operatorname{coth}\left(\sqrt{-\mu}\left(d n-\frac{\sinh (2 d \sqrt{-\mu})}{\Gamma(1+\alpha) \sqrt{-\mu}} t^{\alpha}+\chi\right)\right),
\end{aligned}
$$

where $a_{0}, d, \chi$ and $\mu(<0)$ remain arbitrary. Similarly, setting $C_{1} \neq 0$ and $C_{2}=0$ or $C_{1}=0$ and $C_{2} \neq 0$ in (33), respectively, provides formal periodic wave solutions to Eq. (2) in the form

$$
\begin{aligned}
& u_{n}(t)=a_{0}-\frac{1}{2} \tan (2 d \sqrt{\mu}) \tan \left(\sqrt{\mu}\left(d n-\frac{\sin (2 d \sqrt{\mu})}{\Gamma(1+\alpha) \sqrt{\mu}} t^{\alpha}+\chi\right)\right) \\
& u_{n}(t)=a_{0}+\frac{1}{2} \tan (2 d \sqrt{\mu}) \cot \left(\sqrt{\mu}\left(d n-\frac{\sin (2 d \sqrt{\mu})}{\Gamma(1+\alpha) \sqrt{\mu}} t^{\alpha}+\chi\right)\right) .
\end{aligned}
$$

where $a_{0}, d, \chi$ and $\mu(>0)$ remain arbitrary.

\section{Analysis of Eq. (3)}

To solve Eq. (3), let us first consider the fractional complex transformation

$$
u_{n}=U_{n}\left(\xi_{n}\right), \quad \xi_{n}=d n+\frac{k}{\Gamma(1+\alpha)} t^{\alpha}+\chi,
$$

where $d$ and $k$ are real parameters to be specified, while $\chi$ denotes the phase shift. Substituting (41) into Eq. (3) leads to the equation

$$
k U_{n}^{\prime}\left(U_{n-1}+U_{n}\right)\left(U_{n}+U_{n+1}\right)-2\left(U_{n-1}-U_{n+1}\right) U_{n}^{2}\left(1-U_{n}^{2}\right)=0,
$$

where prime denotes ordinary derivative with respect to the new independent variable $\xi_{n}$. The procedure suggests then to look for special solutions of Eq. (42) in the form

$$
U_{n}=a_{0}+a_{1} \frac{G \prime}{G}, \quad a_{1} \neq 0,
$$

where $G=G\left(\xi_{n}\right)$ satisfies Eq. (15), while $a_{0}$ and $a_{1}$ are arbitrary constants to be specified. From now on, some of the details will be omitted for the sake of brevity.

\subsection{Hyperbolic function solutions}

In case $\mu<0$, substituting (43) and $U_{n \pm 1}$ along with (15) into (42), clearing the denominator, setting the coefficients of $\left(G^{\prime} / G\right)^{l}(l=0,1, \ldots, 6)$ to zero, a system of nonlinear 
algebraic equations for $a_{0}, a_{1}, d, k$ and $\mu$ and is obtained. Two solutions of the resulting system are

$$
\begin{aligned}
& \lambda=0, \quad a_{0}=-\frac{1}{2} \tanh (d \sqrt{-\mu}), \quad a_{1}= \pm \frac{\tanh (d \sqrt{-\mu})}{2 \sqrt{-\mu}}, \\
& k=-\frac{\tanh (d \sqrt{-\mu})}{\sqrt{-\mu}} ; \\
& \lambda=0, \quad a_{0}=\frac{1}{2} \tanh (d \sqrt{-\mu}), \quad a_{1}= \pm \frac{\tanh (d \sqrt{-\mu})}{2 \sqrt{-\mu}}, \\
& k=-\frac{\tanh (d \sqrt{-\mu})}{\sqrt{-\mu}} .
\end{aligned}
$$

Here and henceforth, the signs are ordered in a vertical manner. Setting the parameter values (44) and (45) into expression (43) in accordance with (16) leads to hyperbolic function solutions to Eq. (3) in the form

$$
\begin{aligned}
u_{n}(t)= & -\frac{1}{2} \tanh (d \sqrt{-\mu}) \\
& \pm \frac{1}{2} \tanh (d \sqrt{-\mu}) \frac{C_{1} \cosh \left(\sqrt{-\mu} \xi_{n}\right)+C_{2} \sinh \left(\sqrt{-\mu} \xi_{n}\right)}{C_{1} \sinh \left(\sqrt{-\mu} \xi_{n}\right)+C_{2} \cosh \left(\sqrt{-\mu} \xi_{n}\right)} \\
u_{n}(t)= & \frac{1}{2} \tanh (d \sqrt{-\mu}) \\
& \pm \frac{1}{2} \tanh (d \sqrt{-\mu}) \frac{C_{1} \cosh \left(\sqrt{-\mu} \xi_{n}\right)+C_{2} \sinh \left(\sqrt{-\mu} \xi_{n}\right)}{C_{1} \sinh \left(\sqrt{-\mu} \xi_{n}\right)+C_{2} \cosh \left(\sqrt{-\mu} \xi_{n}\right)},
\end{aligned}
$$

where $\xi_{n}=d n-\tanh (2 d \sqrt{-\mu}) /(\Gamma(1+\alpha) \sqrt{-\mu}) t^{\alpha}+\chi$, while $d, \chi, \mu(<0), C_{1}$ and $\mathrm{C}_{2}$ remain arbitrary.

\subsection{Trigonometric function solutions}

In case $\mu>0$, substituting (46) and $U_{n \pm 1}$ along with (15) into (42), clearing the denominator, setting the coefficients of $\left(G^{\prime} / G\right)^{l}(l=0,1, \ldots, 6)$ to zero, a system of nonlinear algebraic equations for $a_{0}, a_{1}, d, k$ and $\mu$ is obtained. Two solutions of the resulting system are

$$
\begin{array}{llrl}
\lambda=0, & a_{0}=\mp \frac{1}{2} \mathrm{i} \tan (d \sqrt{\mu}), & a_{1}=-\frac{\tan (d \sqrt{\mu})}{2 \sqrt{\mu}}, & k=-\frac{\tan (d \sqrt{\mu})}{\sqrt{\mu}}, \\
\lambda=0, & a_{0}=\mp \frac{1}{2} \mathrm{i} \tan (d \sqrt{\mu}), & a_{1}=\frac{\tan (d \sqrt{\mu})}{2 \sqrt{\mu}}, \quad k=-\frac{\tan (d \sqrt{\mu})}{\sqrt{\mu}} .
\end{array}
$$

Setting the parameter values (48) and (49) into expression (43) in accordance with (17) leads to trigonometric function solutions to Eq. (3) in the form

$$
u_{n}(t)=\mp \frac{1}{2} \mathrm{i} \tan (d \sqrt{\mu})-\frac{1}{2} \tan (d \sqrt{\mu}) \frac{-C_{1} \sin \left(\sqrt{\mu} \xi_{n}\right)+C_{2} \cos \left(\sqrt{\mu} \xi_{n}\right)}{C_{1} \cos \left(\sqrt{\mu} \xi_{n}\right)+C_{2} \sin \left(\sqrt{\mu} \xi_{n}\right)},
$$




$$
u_{n}(t)=\mp \frac{1}{2} \mathrm{i} \tan (d \sqrt{\mu})+\frac{1}{2} \tan (d \sqrt{\mu}) \frac{-C_{1} \sin \left(\sqrt{\mu} \xi_{n}\right)+C_{2} \cos \left(\sqrt{\mu} \xi_{n}\right)}{C_{1} \cos \left(\sqrt{\mu} \xi_{n}\right)+C_{2} \sin \left(\sqrt{\mu} \xi_{n}\right)},
$$

where $\xi_{n}=d n-\tan (2 d \sqrt{\mu}) /(\Gamma(1+\alpha) \sqrt{\mu}) t^{\alpha}+\chi$, while $d, \chi, \mu(>0), C_{1}$ and $C_{2}$ remain arbitrary.

\subsection{Rational function solutions}

In case $\mu=0$, substituting (43) and $U_{n \pm 1}$ along with (15) into (42), clearing the denominator, setting the coefficients of $\left(G^{\prime} / G\right)^{l}(l=2,3, \ldots, 6)$ to zero, a system of nonlinear algebraic equations for $a_{0}, a_{1}, d$ and $k$ is obtained. A solution of the resulting system is

$$
\lambda=0, \quad a_{0}=0, \quad a_{1}= \pm \frac{d}{2}, \quad k=-d,
$$

Inserting the parameter values (52) into expression (43) in accordance with (18) leads to a rational function solution to Eq. (3) in the form

$$
u_{n}(t)= \pm \frac{d}{2} \frac{C_{1}}{C_{1}\left(d n-\frac{d}{\Gamma(1+\alpha)} t^{\alpha}+\chi\right)+C_{2}}
$$

where $d, \chi, C_{1}$ and $C_{2}$ remain arbitrary.

\section{Discussion and conclusion}

It is worth to comment about some other powerful techniques which have been proven to be useful in solving problems of nonlinear science. Recently, the discrete Jacobi subequation method was presented in [34] for solving nonlinear DDEs via Jacobi elliptic functions sn, cn and dn. More lately, the superposition principle was successfully used to construct exponential traveling wave solutions to bilinear equations [23]. Another interesting technique is the Frobenius integrable decompositions method [22] for nonlinear PDEs. The main idea of this method is to convert nonlinear PDEs into systems of Frobenius integrable ODEs. Based on symbolic computation systems, the transformed rational function method [21] and the multiple exp-function method [24] was also introduced for presenting single/multiple wave solutions. The main point of the transformed rational function method is to search for solutions of rational type to variable-coefficient ODEs deduced from given PDEs. The multiple exp-function method is more general than the $\left(G^{\prime} / G\right)$-expansion method (and it generates all kinds of exact solutions such as solitons, positons and complexitons). Besides, the ansatze method [20] uses the idea of separating nonlinear PDES into integrable ODEs. The crucial point of the ansatze method is to consider a simplest equation which is solvable by quadratures. For example, more general solutions to PDEs could be generated using the general Ricatti equation whose general solutions (40), (41), and (42) can be found in [20]. Our work can be related to the just-mentioned sophisticated methods in the sense that they might provide more interesting results than ours. For convenience, we shall reserve this idea for another study. 
In this paper, DDEs of rational type are extended to fractional order via an improved version of the $\left(G^{\prime} / G\right)$-expansion method. In the related literature, research focusing on fractional DDEs of rational type does not exist. Hence, the present study has made an attempt to fill the gap in the related literature. Besides, it is significant in that it has provided insight to the analytic investigation of fractional DDEs of rational type. Two time-fractional DDEs according to the Jumarie's modified Riemann-Liouville derivative are analyzed in a straightforward way. The availability of symbolic computation systems (such as MATHEMATICA, MATLAB and MAPLE) has made a valuable contribution to the present study. As a result, three types of exact solutions are derived: hyperbolic, trigonometric and rational. The present study also confirms that the fractional complex transformation is extremely useful for converting FDEs to ODEs. The fractional complex transformation corresponds to only wave solutions for FDEs. It is observed that the obtained solutions (50) and (51) are complex-valued, namely, mathematically exist but physically unrealistic. Of course, some classes of DDEs of fractional order may not be suited for the presented technique. For instance, consider the following equation:

$$
D_{t}^{\alpha} u_{n}=\frac{\left(u_{n-1}-u_{n+1}\right)\left(1-u_{n}^{2}\right)\left(\eta^{2}-\mu^{2} u_{n}^{2}\right)}{\left(u_{n-1}+u_{n}\right)\left(u_{n}+u_{n+1}\right)}, \quad 0<\alpha \leqslant 1,
$$

where $\eta$ and $\mu$ are assumed to be arbitrary parameters. A limitation of the present study is that due to the method used only complex-valued solutions for Eq. (54) can be extracted. The problem of finding real-valued solutions (if exist) for Eq. (54) remains as open research problem. The case $\alpha=1$ of Eq. (54) is considered in [29]. It can be concluded that the $\left(G^{\prime} / G\right)$-expansion method is an effective method to find exact solutions to fractional DDEs.

Acknowledgments. The author is grateful to the anonymous referees for careful reading and constructive comments.

\section{References}

1. İ. Aslan, A note on the $\left(G^{\prime} / G\right)$-expansion method again, Appl. Math. Comput., 217:937-938, 2010.

2. Z. Bin, $\left(G^{\prime} / G\right)$-expansion method for solving fractional partial differential equations in the theory of mathematical physics, Commun. Theor. Phys., 58:623-630, 2012.

3. J. Cresson, Non-differentiable variational principles, J. Math. Anal. Appl., 307:48-64, 2005.

4. M. Cui, Compact finite difference method for the fractional diffusion equation, J. Comput. Phys., 228:7792-7804, 2009.

5. C.Q. Dai, Y.Y. Wang, Exact traveling wave solutions of the discrete nonlinear Schrödinger equation and the hybrid lattice equation obtained via the exp-function method, Phys. Scr., 78 , 015013, 2008.

6. A.M.A. El-Sayed, M. Gaber, The Adomian decomposition method for solving partial differential equations of fractal order in finite domains, Phys. Lett. A, 359:175-182, 2006. 
7. E. Fermi, J. Pasta, S. Ulam, Collected Papers of Enrico Fermi, Univ. of Chicago Press, Chicago, 1965.

8. K.A. Gepreel, S. Omran, Exact solutions for nonlinear partial fractional differential equations, Chin. Phys. B, 21, 110204, 2012.

9. J.H. He, A coupling method of a homotopy technique and a perturbation technique for nonlinear problems, Int. J. Nonlinear Mech., 35:37-43, 2000

10. J.H. He, G.C. Wu, F. Austin, The variational iteration method which should be followed, Nonl. Sci. Lett. A, 1:1-30, 2010.

11. X.B. Hu, W.X. Ma, Application of Hirota's bilinear formalism to the Toeplitz lattice-some special soliton-like solutions, Phys. Lett. A, 293:161-165, 2002.

12. Q. Huang, G. Huang, H. Zhan, A finite element solution for the fractional advection-dispersion equation, Adv. Water Resour., 31:1578-1589, 2008.

13. G. Jumarie, Modified Riemann-Liouville derivative and fractional Taylor series of nondifferentiable functions further results, Comput. Math. Appl., 51:1367-1376, 2006.

14. G. Jumarie, New stochastic fractional models for Malthusian growth, the Poissonian birth process and optimal management of populations, Math. Comput. Modelling, 44:231-254, 2006.

15. G. Jumarie, Laplace's transform of fractional order via the Mittag-Leffler function and modified Riemann-Liouville derivative, Appl. Math. Lett., 22:1659-1664, 2009.

16. A.A. Kilbas, H.M. Srivastava, J.J. Trujillo, Theory and Applications of Fractional Differential Equations, Elsevier, San Diego, 2006.

17. K.M. Kolwankar, A.D. Gangal, Local fractional Fokker-Planck equation, Phys. Rev. Lett., 80:214-217, 1998.

18. Z.B. Li, J.H. He, Fractional complex transformation for fractional differential equations, Math Comput. Appl., 15:970-973, 2010.

19. B. Lu, The first integral method for some time fractional differential equations, J. Math. Anal. Appl., 395:684-693, 2012.

20. W.X. Ma, B. Fuchssteiner, Explicit and exact solutions to a Kolmogorov-Petrovskii-Piskunov equation, Int. J. Nonlinear Mech., 31:329-338, 1996.

21. W.X. Ma, J.H. Lee, A transformed rational function method and exact solutions to the $3+1$ dimensional Jimbo-Miwa equation, Chaos Solitons Fractals, 42:1356-1363, 2009.

22. W.X. Ma, H. Wu, J. He, Partial differential equations possessing Frobenius integrable decompositions, Phys. Lett. A, 364:29-32, 2007.

23. W.X. Ma, Y. Zhang, Y. Tang, J. Tu, Hirota bilinear equations with linear subspaces of solutions, Appl. Math. Comput., 218:7174-7183, 2012.

24. W.X. Ma, Z. Zhu, Solving the $(3+1)$-dimensional generalized KP and BKP equations by the multiple exp-function algorithm, Appl. Math. Comput., 218:11871-11879, 2012.

25. W.X. Ma, Y. You, Rational solutions of the Toda lattice equation in Casoratian form, Chaos Solitons Fractals, 22:395-406, 2004. 
26. K.S. Miller, B. Ross, An Introduction to the Fractional Calculus and Fractional Differential Equations, Wiley, New York, 1993.

27. K. Narita, New discrete modified KdV equation, Prog. Theor. Phys., 86:817-824, 1991.

28. K. Narita, Special solutions to nonlinear difference-differential equations, J. Math. Anal. Appl., 205:273-279, 1997.

29. K. Narita, Solutions for a system of difference-differential equations related to the self-dual network equation, Prog. Theor. Phys., 106:1079-1096, 2001.

30. Z. Odibat, S. Momani, A generalized differential transform method for linear partial differential equations of fractional order, Appl. Math. Lett., 21:194-199, 2008.

31. Z. Odibat, S. Momani, The variational iteration method: An efficient scheme for handling fractional partial differential equations in fluid mechanics, Comput. Math. Appl., 58:2199_ $2208,2009$.

32. I. Podlubny, Fractional Differential Equations, Academic Press, San Diego, 1999.

33. M. Toda, Theory of Nonlinear Lattices, Springer-Verlag, New York, 1989.

34. Z. Wang, W.X. Ma, Discrete Jacobi sub-equation method for nonlinear differential-difference equations, Math. Methods Appl. Sci., 33:1463-1472, 2010.

35. G.C. Wu, E.W.M. Lee, Fractional variational iteration method and its application, Phys. Lett. A, 374:273-279, 2010.

36. P. Yang, Y. Chen, Z.B. Li, ADM-Padé technique for the nonlinear lattice equations, Appl. Math. Comput., 210:362-375, 2009.

37. S. Zhang, L. Dong, J.M. Ba, Y.N. Sun, The $\left(G^{\prime} / G\right)$-expansion method for nonlinear differential-difference equations, Phys. Lett. A, 373:905-910, 2009.

38. S. Zhang, L. Dong, J.M. Ba, Y.N. Sun, The $\left(G^{\prime} / G\right)$-expansion method for a discrete nonlinear Schrödinger equation, Pramana-J. Phys., 74:207-218, 2010.

39. S. Zhang, H.Q. Zhang, Fractional sub-equation method and its applications to nonlinear fractional PDEs, Phys. Lett. A, 375:1069-1073, 2011.

40. S.D. Zhu, Y.M. Chu, S.L. Qiu, The homotopy perturbation method for discontinued problems arising in nanotechnology, Comput. Math. Appl., 58:2398-2401, 2009. 